\title{
Valproate-Induced Reversible Atrophy and Cognitive Decline:
} a case report

\author{
Emily Innes ${ }^{1}$, Alexandra M Johnson ${ }^{2}$ \\ ${ }_{1}^{1 B A p p S c i(P h t y)}$ (Hons), MBBS (Hons); Paediatric Registrar, Sydney Children's Hospital, Sydney, Australia; Adjunct Lecturer, \\ University of Notre Dame, Sydney; Australia. Emily.innes@health.nsw.gov.au \\ 2MBBS BSc(Med), MMed; Department of Neurology, Sydney Children's Hospital, Sydney, Australia; University of New South Wales, \\ Sydney, Australia; Alexandra.johnson@health.nsw.gov.au
}

Corresponding author: Dr Alexandra Johnson; Neurology Department Sydney Children's Hospital, High St, Randwick NSW Australia 2031; Fax: +61 (2) 9382 1580; Phone: +61 (2) 9382 1111; Email: Alexandra.johnson@health.nsw.gov.au

\section{ABSTRACT}

Background: Valproate (VPA) has been previously described to cause reversible cerebral atrophy and cognitive decline, but few cases are reported, and neuropsychological data is lacking. We report a case of VPA-induced encephalopathy in an 11-year-old girl with temporal lobe epilepsy, presenting with impaired cognition and sedation combined with cerebral atrophy.

Methods: Cognitive capacity was assessed using Wechsler Intelligence Scales for Children IV (WISC-IV), nonstandardised word lists, and visual reproduction. Brain MRI was performed prior to, during, and post VPA therapy.

Results: Our patient demonstrated average full-scale intelligence quotient (IQ), verbal comprehension, perceptual reasoning and working memory. There was a marked discrepancy in processing speed, which ranked low average (score - 80; 9th percentile). Difficulties with mental abstraction and manipulation were noted. Brain MRI (3T) demonstrated mild generalised parenchymal atrophy. Cessation of VPA resulted in dramatic improvement in clinical symptoms (one month after cessation) and normalisation of brain MRI (11 months after cessation). Progress neuropsychological testing 13 months after cessation showed marked improvements in processing speed.

Conclusion: This case provides an essential reminder of this rare but importantly reversible syndrome with new information supporting the neuropsychological changes involved.

Keywords: Valproate, encephalopathy, atrophy, cognition

(c) 2018 Innes \& Johnson; licensee JICNA

\section{BACKGROUND}

Sodium valproate (VPA) is a widely used antiepileptic for the treatment of paediatric epilepsy. A rarely described serious adverse reaction to VPA therapy is a reversible encephalopathy, characterised by cognitive regression and behavioural disturbances associated with cerebral atrophy. With consent, we report a case of VPA-induced reversible cerebral atrophy in an 11-year-old girl with temporal lobe epilepsy. A search of the literature conducted using PubMed and OVID databases with search terms "valproate", "atrophy" and "encephalopathy" identified six case reports of VPA-induced encephalopathy from 1987-2014. As literature is sparse, all cases were reviewed with acknowledgement of the bias associated with time and case reporting. Neuropsychological changes measured by standardised testing were reported in four cases. No data has been presented in the last ten years. This case report provides further detail describing neuropsychological changes. Predominant clinical findings include markedly reduced alertness, impaired processing speed, and manipulation of verbal information.

\section{CASE REPORT}

The patient presented aged seven years with focal seizures with left temporal epileptiform activity as well as generalised discharges noted on electroencephalogram (EEG). VPA was commenced as the initial anti-epileptic. Her epilepsy proved resistant to medication as seizure freedom was not obtained despite increasing VPA dose, with subsequent trials of adjunctive carbamazepine (ceased due to rash), levetiracetam and clobazam.

Initial MRI brain prior to VPA therapy demonstrated no signal or structural abnormality (Figure 1A). A cerebral flurodeoxyglucose positron emission tomography confirmed left temporal lobe hypometabolism. At age 9 years 4 months drowsiness, reduced concentration and memory difficulties were noted, particularly affecting school performance in mathematics and spelling. A gradual decline in alertness and cognition continued over the next year, and it was recognised this was out of keeping with the usual effects of polypharmacy. 
Brain MRI was repeated at age 10 years 5 months. Although initially reported as normal, the brain MRI (3T) demonstrated mild generalised parenchymal atrophy and ventricular enlargement (Figure 1B). At the time, VPA level was high at $854 \mathrm{umol} / \mathrm{L}(350-700)$ on $38 \mathrm{mg} / \mathrm{kg} / \mathrm{day}$ VPA. Serum examination was otherwise unremarkable. As VPA-induced encephalopathy was suspected, the medication was ceased as a primary intervention, rather than investigate for metabolic and/or genetic causes of generalised atrophy in the context of epilepsy.

Results

Cognitive capacity was assessed using Wechsler Intelligence Scales for Children IV (WISC-IV), non-standardised word lists, and visual reproduction (see Table 1). At the time of the clinical symptoms, full-scale intelligence quotient (IQ), verbal comprehension, perceptual reasoning, and working memory were ranked average. A significant discrepancy was noted in terms of her processing speed, which ranked low-average (score - 80; 9th percentile). Neuropsychological examination reported difficulties with mental manipulation and abstraction within the verbal domain, and reduced capacity to manipulate verbal information in memory. It was assumed that this would specifically impair her performance in subjects such as mathematics and spelling.

VPA was ceased with dramatic improvement in clinical symptoms within one month. Oxcarbazepine was trialled with a pleasing reduction in seizure burden. She currently has rare auras (monthly) and is maintained on oxcarbazepine monotherapy.

At 11 months post VPA cessation, brain MRI had returned to normal (Figure 1C). Progress neuropsychological testing 13 months after cessation showed a marked improvement in processing speed, which has now ranked in the average range (score - 97; 42nd percentile) (Table 1). Verbal comprehension index advanced from average to high-average, and full-scale IQ increased from 47th to 63rd percentile.

Discussion

This case further describes a form of VPA-induced encephalopathy characterised by cerebral atrophy and cognitive decline. Previously reported clinical manifestations include: (1) cognitive regression including a deterioration in baseline IQ [1-5]; (2) impaired memory and concentration [14]; (3) a reduced level of awareness from apathy [2-3] to a stuporous coma [1]; (4) a behavioural disturbance including emotional lability [3-4] and developmental regression [3]; and (5) occasionally motor signs including tremor, dysarthria and ataxia [1-3]. Motor signs were normal and did not evolve in this case.

Reversibility of both clinical manifestations and cerebral atrophy are hallmark features and are diagnostic. Baseline function and intelligence rapidly return over the course of weeks to months [1-5]. Restoration of the normal brain anatomy on computed tomography/MRI lags clinical improvement, yet is achieved between one to two years, as occurred in this case $[1-3,5]$.

Previous reports of VPA-induced encephalopathy have demonstrated reduction in full-scale IQ and verbal comprehension index [1-4]. In this case, verbal skills, memory, and processing speed were particularly affected. Previous reports of children with deficits in working memory (particularly phonological and complex working memory) show concomitant language deficits and impaired comprehension of language and mathematics [6]. Our case did display difficulty with these areas: mathematics, spelling, and impaired manipulation of verbal information in memory. Processing speed has not been significantly impaired in other reports of VPA-induced encephalopathy, though this was a striking feature of both impairment and then improvement in this case. This case provides further information regarding cognitive features potentially impacted in VPA-induced atrophy and may help other clinicians to distinguish VPA-associated cognitive decline from another new or previously unsuspected pathological process.

The presence of cerebral atrophy, often a sign of a neurodegenerative process, prompts clinicians to investigate for an underlying neurometabolic process. In the setting of generalised atrophy and altered cognition or awareness, VPA-induced encephalopathy is an important differential due to its iatrogenic nature and its reversibility. It is important to recognise this entity to potentially reduce the burden of unnecessary testing with associated costs and familial anxiety, and to shorten clinical symptomatology affecting the child.

Pathophysiology of VPA-induced encephalopathy is not well elicited, yet there is sufficient evidence excluding metabolic and neurodegenerative causation. Previous cases have reported extensive normal neurometabolic testing: plasma lactate, pyruvate, ammonia, copper/caeruloplasmin, acylcarnitines, very-long chain fatty acids, amino acids, organic acids and lysosomal enzymes [1,3,4,7]; cerebrospinal fluid glucose, protein, lactate and pyruvate $[1,3,7]$; urine reducing substances, organic acids and specific amino acids $[3,4,7]$. MRI spectroscopy performed in one case demonstrated a normal lactate peak and decreased $\mathrm{N}$-Acetylaspartate to creatine ratio [7]. EEG recordings have demonstrated a normal background rhythm with interictal epileptic features that are unchanged from baseline [1-5].

From a pharmacological viewpoint, there is evidence this is not a dose-dependent or toxic encephalopathy, as previous cases note therapeutic VPA levels [1-5]. The underlying pathological processes that occur in VPA-induced encephalopathy with atrophy and cognitive decline remain unclear. Underlying mechanisms that have been proposed include inhibited neuronal outgrowth [8], -aminobutyric acid (GABA) mediated dopamine inhibition [9] and a mitochondrial mutation affecting the MT-ATP8 gene [10]. Overall, it is recommended that investigations are limited and VPA discontinued as a priority.

\section{CONCLUSION}

VPA-induced encephalopathy is rare but reversible, and is therefore important to recognise. Investigations should be limited as discontinuation of VPA is advocated as primary intervention. This case adds further information regarding the neuropsychological profile of patients with VPA-induced encephalopathy, with verbal skills, memory and processing speed particularly affected.

\section{Abbreviations}

EEG - Electroencephalogram

GABA - $\mathrm{y}$-aminobutyric acid

$I Q$ - Intelligence quotient

VPA - Sodium valproate

WISC-IV - Wechsler Intelligence Scales for Children IV 


\section{Acknowledgments}

We would like to thank our patient and her family for their support.

\section{Competing interests}

The authors have declared that no competing interest exists.

\section{Author contributions}

AJ contributed through data collection, interpretation and manuscript revisions. El was primarily involved in the development of the manuscript as well as data interpretation.
This is an Open Access article distributed under the terms of the Creative Commons Attribution License (http://creativecommons.org/ licenses/by/4.0), which permits unrestricted use, distribution, and reproduction in any medium, provided the original work is properly credited. The Creative Commons Public Domain Dedication waiver (http://creativecommons.org/publicdomain/zero/1.0/) applies to the data made available in this article, unless otherwise stated.

\section{Cite this article as:}

Innes \& Johnson: Valproate-Induced Reversible Atrophy and Cognitive Decline: a case report, JICNA 2018 18:118

\section{REFERENCES}

1. Straussberg R, Kivity S, Weitz R, Harel L, Gadoth N: Reversible cortical atrophy and cognitive decline induced by valproic acid. European Journal of Paediatric Neurology. 1998; 2:213-218. doi:10.1016/S10903798(98)80022-6

2. Guerrini R, Belmonte A, Canapicchi R, Casalini C, Perucca E: Reversible pseudoatrophy of the brain and mental deterioration associated with valproate treatment. Epilepsia. 1998; 39(1):27-32. doi:10.1111/j.1528-1157.1998.tb01270.x

3. Papazian O, Canizales E, Alfonso I, Archilia R, Duchowny M, Aicardi J: Reversible dementia and apparent brain atrophy during valproate therapy. Annals of Neurology. 1995 Oct; 38(4):687-691. doi: 10.1002/ana.410380423

4. Galimberti C, Diegoli M, Sartori I, Uggetti C, Brega A, Tartara A, et al: Brain pseudoatrophy and mental regression on valproate and a mitochondrial DNA mutation. Neurology. 2006 Nov; 67:1715-1717. doi:10.1212/01. wnl.0000242882.58086.9a

5. Mclachlan, R: Pseudoatrophy of the brain with valproic acid monotherapy. The Canadian Journal of Neurological Sciences. 1987; 14:294-296.
6. Gathercole S: Cognitive approaches to the development of short-term memory. Trends in Cognitive Sciences. 1999 Nov; 3(11):410-419. doi:10.1016/S13646613(99)01388-1

7. Lovett M, Skidmore D, Mohamed I: Valproate-induced pseudoatrophy: expanding the clinical and imaging spectrum. Pediatric Neurology. 2014; 51:284-285. doi:10.1016/j.pediatrneurol.2014.04.019

8. Qian Y, Zheng Y, Tiffany-Castiglioni E: Valproate reversibly reduces neurite outgrowth by human SY5Y neuroblastoma cells. Brain Research. 2009 Nov; 1302:21-33. doi: 10.1016/j.brainres.2009.09.051

9. Gale K: Role of the substantia nigra in GABA-mediated anticonvulsant actions. Advances in Neurology. 1986; 44:343-364.

10. De Michele G, Sorrentino P, Nesti C, et al. Reversible Valproate-Induced Subacute Encephalopathy Associated With a MT-ATP8 Variant in the Mitochondrial Genome. Front Neurol. 2018;9:728. 


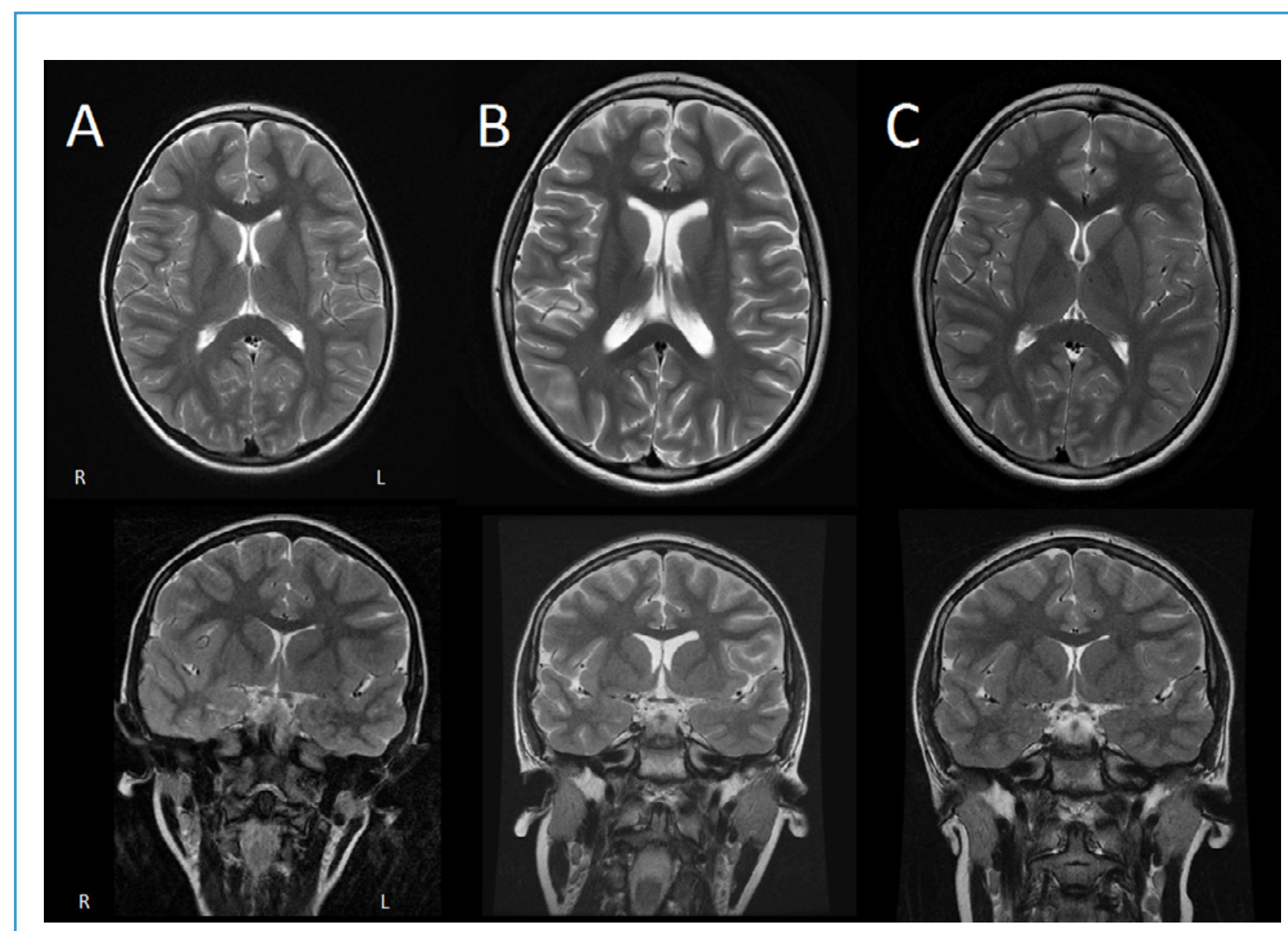

Figure 1 - Brain MRI

(A) Baseline MRI prior to VPA showing normal T2 axial and coronal views. (B) Increased size of ventricles with rounding of horns and increased sulcal space indicative of cerebral atrophy. (C) Recovery after 11 months off VPA with normalisation of ventricular size and cerebral sulci. 
Table 1 - Comparison of Wechsler Intelligence Scales for Children IV (WISC-IV) global intelligence during and post VPA therapy

\begin{tabular}{|c|c|c|c|c|c|c|}
\hline \multirow{2}{*}{ WISC-IV } & \multicolumn{3}{|c|}{ During VPA therapy } & \multicolumn{3}{|c|}{ Post VPA cessation } \\
\hline & Score & Percentile & Rank & Score & Percentile & Rank \\
\hline Verbal Comprehension Index & 108 & 70th & average & 114 & $82 \mathrm{nd}$ & high average \\
\hline Perceptual Reasoning Index & 105 & 55th & average & 102 & 55th & average \\
\hline Working Memory Index & 97 & 42nd & average & 94 & 34th & average \\
\hline Processing Speed Index & 80 & 9th & low average & 97 & 42nd & average \\
\hline Full-scale IQ & 99 & 47th & average & 106 & $63 r d$ & average \\
\hline
\end{tabular}

